\title{
Non-Uniform Sinusoidally Modulated Half-Mode Leaky-Wave Lines for Near-Field Focusing Pattern Synthesis
}

\author{
Alejandro Javier Martinez-Ros, Student Member, IEEE, José Luis Gómez-Tornero, Member, IEEE, \\ Vicente Losada, Francisco Mesa, Fellow, IEEE, and Francisco Medina, Fellow, IEEE
}

\begin{abstract}
A novel non-uniform sinusoidally modulated half-mode microstrip structure with application to near-field focused leaky-wave radiation in the backward Fresnel zone is proposed. First, it is presented a dispersion analysis of the constituent backward leaky wave in the sinusoidally modulated unit cell in half-width microstrip technology. This information is then used to design a finite non-uniform line that focuses the radiated fields at the desired point. Finally, eight similar line sources are arranged in a radial array to generate a three-dimensional focused spot located at the desired focal length over the simple central coaxial feeding. Simulated and experimental results are presented to validate the proposed simple approach.
\end{abstract}

Index Terms-Backward radiation, half-mode leaky-wave antennas, near-field focusing, microwave lens, sinusoidal modulation, tapered leaky mode.

\section{INTRODUCTION}

$\mathbf{S}$ INUSOIDALLY modulated (SM) reactance surfaces were first proposed by Oliner and Hessel in 1959 as an elegant and canonical way to control the propagation of surface and leaky waves in periodic transmission media [1]. In this seminal theoretical work it was assumed a sinusoidal variation of the equivalent surface reactance along the propagation direction that was soon applied to a variety of practical cases [2]-[7]. Recently, renewed interest is given to SM circuits in printed/planar technology due to their ability to control the propagation and radiation properties in low-cost artificial planar guides, arising

\footnotetext{
Manuscript received June 20, 2014; revised December 09, 2014; accepted December 11, 2014. Date of publication December 29, 2014; date of current version March 02, 2015. This work was supported in part by the Ministerio de Educación y Ciencia Español and FEDER, Refs. TEC2013-47037-C5-5-R, TEC2010-21520-C04-04 and TEC2013-41913-P, by the ESF in the framework of the RNP-NEWFOCUS, and in part by the Spanish Regional Seneca project 08833/PI/08

A. J. Martinez-Ros and J. L. Gómez-Tornero are with the Department of Communication and Information Technologies, Universidad Politécnica de Cartagena, Cartagena 30202 Spain (e-mail: alejandro.martinez@upct.es, josel.gomez@upct.es).

V. Losada and F. Mesa are with the Microwaves Group, Department of Applied Physics 1, Escuela Técnica Superior de Ingeniería Informática, University of Seville, 41012-Seville, Spain (e-mail: losada@us.es; mesa@us.es).

F. Medina is with the Faculty of Physics, Department of Electronics and Electromagnetism, University of Seville, 41012-Seville, Spain (e-mail: medina@us. es).

Color versions of one or more of the figures in this paper are available online at http://ieeexplore.ieee.org.

Digital Object Identifier 10.1109/TAP.2014.2386339
}

the area of planar metamaterial dispersion engineering, also referred to as metasurfing [8]-[10]. In this way, sinusoidally and linearly-chirped width-modulated microstrip lines have been proposed for enhanced control of the stopbands in microwave filters [11], [12], and to synthesize dispersive linear group delays with application to real-time analog signal processing [13]. On the other hand, printed SM metasurfaces have also been proposed to control the radiation from leaky waves with application to directive holographic antennas [14]-[23]. In all cases, it is essential to determine how to control the guiding and radiation characteristics of the constituent surface and leaky modes for each selected metasurface technology.

Some previous designs based on sinusoidally modulated reactance surfaces [1], [19], [20], sinusoidally modulated material parameters [12], [21], [22], metamaterial transmission lines [24] as well as periodic half-mode leaky-wave antennas (HMLWAs) [25]-[27] have shown the capability to radiate backward and to perform certain control over the phase or the amplitude of the radiated fields. As is well known [1], if the period of the structure is adequately chosen, the backward radiation comes from the existence of a backward leaky wave (negative scanning angle $\theta_{\mathrm{RAD}}$ ) associated with the $n=-1$ space harmonic [the rest of harmonics are in the invisible spectrum (non radiating)]. The constant value (along the propagation direction) of the leaky-wave phase constant and leakage rate of a uniform SM LWA generates a linear-phase and exponential-amplitude aperture distribution that results in a plane-wave focusing at a given scanning angle $\theta_{\mathrm{RAD}}$ in the far-field zone (all sections of the LWA radiate with the same angle $\theta_{\mathrm{RAD}}$ ). Although in some previous works, e.g., [21], [22], non-uniform structures have been used in order to obtain impedance matching and a smoother transition between the bound and the leaky wave regions, no control over both the phase constant and the leakage rate of the antenna has been reported so far to achieve a tapered illumination function (both in amplitude and phase) along the antenna length. As a result, all reported holographic SM microstrip LWAs [14]-[23] only provide scanned beams focused in the far field.

In this work, a simultaneous control of both the phase constant and the leakage rate is employed to design a tapered illumination function, which is applied to obtain near-field focusing from an array of 1-D leaky-wave antennas (LWAs). Specifically, we propose the use of non-uniform (NU) SM LWAs to generate arbitrary aperture field distributions (amplitude and phase) 


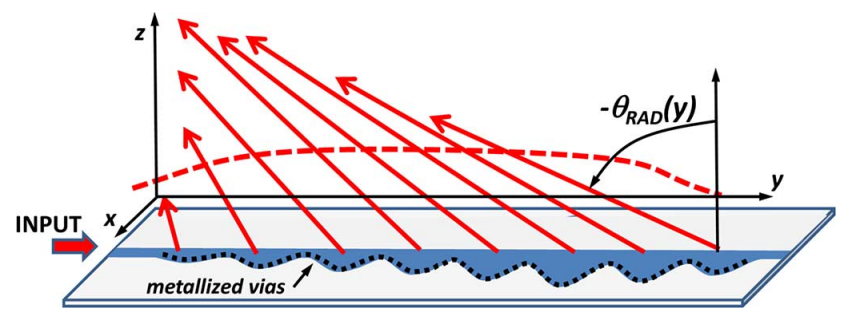

Fig. 1. Scheme of the sinusoidally modulated HMLWA with a non-uniform taper performed to control the backward radiated waves both in amplitude and phase.

that can synthesize near-field focused beams in the backward quadrant, as sketched in Fig. 1. To this aim, the uniform SM topology must be modified so that non-uniform SM unit cells scatter the electromagnetic waves with a defined non-uniform negative phase delay in order to produce constructive interference at the desired point in the Fresnel zone, and with equalized amplitude for optimum aperture efficiency and side-lobe level. In particular, it is considered the case of NU SM microstrip half-width LWAs (HWLWAs) to taper the amplitude and phase of backward radiating leaky waves. The necessity of such tapering is illustrated with the design of a practical leaky-wave lens focused in the backward quadrant, which can thus be arranged in a radial-array fashion to obtain 3-D focusing using a simple central feeding as proposed in [28]. In this manner, the resulting structure can avoid more complex feeding networks as the one proposed in [29], whose radiating elements can only be designed to operate in the forward quadrant and hence cannot be fed from a common single input point. Moreover, it is also important to note that other proposed designs based on sinusoidal modulation of waveguide boundary conditions as [2]-[5], can also theoretically radiate backward and provide simultaneous control on the leakage rate and phase constant, and they can even be arranged in a radial array fashion to provide 3-D near-field focusing. However, compared to these designs based on modulation of bulky waveguides, sinusoidally modulated printed circuits as [6]-[24] provide planar designs which allow for a PCB integration. As a result, the structure proposed in this paper, which is based on sinusoidally modulated half-mode microstrip lines, provides a lower profile and more compact design than waveguide-based solutions [2]-[5].

The paper is distributed as follows: Section II presents the dispersion analysis of the proposed SM half-width microstrip line and the design of the NU SM HWLWA to focus at a desired point in the backward region. Then, a central-fed radial-array of such NU SM HWLWAs is described in Section III to produce a 3-D near-field focused planar microwave lens. Section IV reports the experimental results, showing the ability to create a 3-D focused beam at the desired location with the expected focal depth and focal width, and demonstrating the utility of NU SM microstrip lines for that purpose. Finally, the conclusions of the paper are summarized in Section V.

\section{ANAlysis OF THE SinUSOIDALly MOdUlated HMLWA}

With the aim of obtaining a radiating element with capability to radiate backward and with flexibility to control its complex illumination function, a conventional HMLWA with a sinusoidal modulation is proposed. The HMLWA is based on a microstrip line with one of its sides short-circuited by a perfect electric conductor (PEC) (see top view in Fig. 2(a)). Thus, the first higher order leaky mode of the conventional microstrip line becomes the fundamental one in the HMLWA [30], [31], whose electric field distribution is horizontally polarized along the $E$-plane ( $x z$-plane), as shown in Fig. 2(c). The complex wavenumber of this leaky mode can be expressed as

$$
k_{y}(y)=\beta_{y}(y)-j \alpha_{y}(y)
$$

with $\beta_{y}$ being the phase constant and $\alpha_{y}$ the radiation rate, which are in general dependent on the local source coordinate $(y)$ along the LWA radiating length. From the value of $\beta_{y}$ and its relation with the free-space wavenumber $k_{0}$, the radiation angle of the leaky mode can be determined [32] (assuming $\alpha_{y} \ll \beta_{y}$ ) as

$$
\sin \theta_{\mathrm{RAD}}(y) \approx \beta_{y}(y) / k_{0}
$$

As it is observed in (2), the angle $\theta_{\mathrm{RAD}}$ takes positive values as long as $\beta_{y}>0$. However, in the case of periodic structures with period $P$, the phase constant $\beta_{y n}$ of each space harmonic ( $\mathrm{SH}$ ) comprising the Floquet expansion of the fields is given by

$$
\beta_{y n}(y)=\beta_{y}(y)+\frac{2 n \pi}{P}
$$

and thus each $n$-th $\mathrm{SH}$ could radiate with a different angle given by

$$
\sin \theta_{\mathrm{RAD}_{n}}(y) \approx \frac{\beta_{y n}(y)}{k_{0}}=\frac{\beta_{y}(y)}{k_{0}}+\frac{2 n \pi}{k_{0} P} .
$$

Therefore, a suitable choice of $P$ can make that some of the $n=$ $-1,-2, \ldots$ space harmonics radiate backward $\left(\left|\beta_{y n} / k_{0}\right|<1\right)$ with the angle defined by (4) while the rest of harmonics lie in the slow-wave regime $\left(\left|\beta_{y n} / k_{0}\right|>1\right)$ and do not contribute to leaky radiation. Typically, only the $n=-1$ harmonic is desired for radiation, and this single-harmonic backward radiation condition can be satisfied provided that $\lambda_{0} / 2<P<\lambda_{0}$ [1].

According to the previous discussion, the conventional HMLWA (see Fig. 2(a)) can be modulated with a periodic function of period $P$ in order to make it radiate only with the $n=-1$ space harmonic. To this aim, the strip width $W$ is locally modulated along the antenna length by the following sinusoidal function:

$$
W(y)=W_{m}(y)\left[1+M(y) \sin \left(\frac{2 \pi}{P} y\right)\right]
$$

where $W_{m}(y)$ is the average value of the strip width $W(y)$ at the longitudinal position $y$, and $M(y)$ is the modulation index of the sine function. The resulting sinusoidally modulated HMLWA is shown in Fig. 2(b), where it can be seen the sinusoidal variation that has been performed along the PEC wall side. 


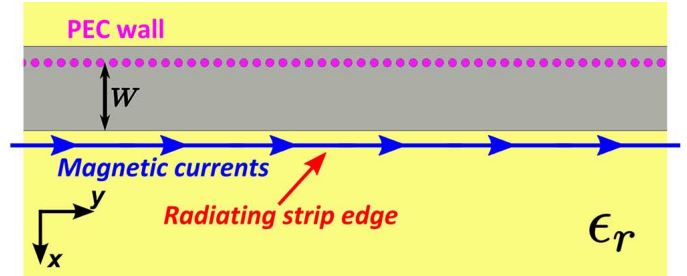

(a)

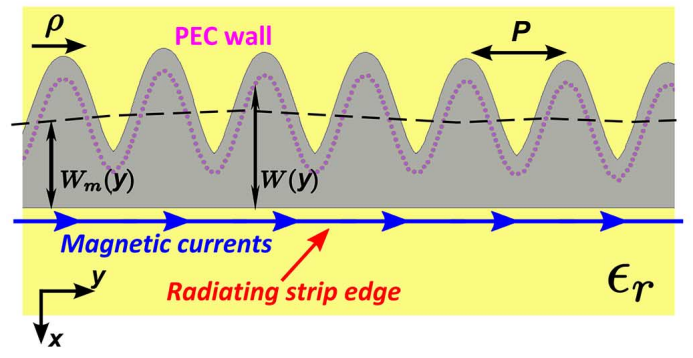

(b)

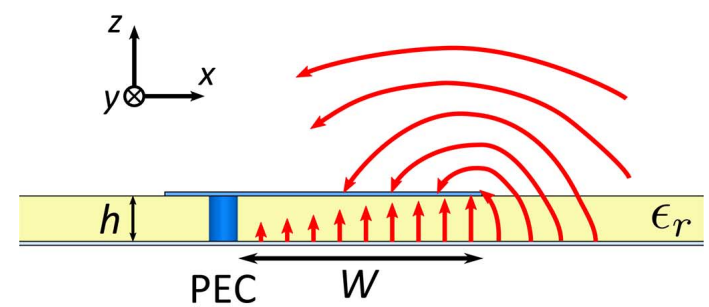

(c)

Fig. 2. Top view for two different variations of the HMLWA with their main geometrical parameters: (a) conventional HMLWA, (b) sinusoidally modulated HMLWA, and (c) electric field lines at the cross section of the HMLWA (E-plane).

It is important to note that in order to design a tapered illumination function along the HMLWA length, the radiated fields have to be properly controlled both in phase and amplitude [33]. Thus, the sinusoidal modulation of the HMLWA width can no longer be uniform but must be non-uniform. Specifically, the average strip width $W_{m}$ is varied to control the phase constant of the fundamental mode $\beta_{y}$ and to avoid that it falls within the fast-wave region $\left(\beta_{y} / k_{0}<1\right)$, while the uniform period $P \gtrsim \lambda_{0} / 2$ provides backward radiation from the $n=-1 \mathrm{SH}\left(\left|\beta_{y_{-1}} / k_{0}\right|<1\right)$. Due to the periodic modulation of the structure a set of space harmonics $(\mathrm{SH})$ can be excited. Hence, it is important to keep unwanted $\mathrm{SH}$ away from the fast-wave region $\left(\left|\beta_{n} / k_{0}\right|<1\right)$ to avoid any spurious radiation. To this aim, the strip width, $W_{m}$, is set to a value larger than $5 \mathrm{~mm}$ in order to avoid the radiation from the fundamental $n=0 \mathrm{SH}\left(\beta_{0} / k_{0}>1\right)$. Moreover, the chosen size of the fundamental period of the structure $(P=11.5 \mathrm{~mm}$ $\left.\approx \lambda_{0} / 2\right)$ ensures that the rest of the $\mathrm{SH}(n=-2,-3, \ldots)$ are out of the fast-wave region in all cases. This is the result of their relation with the fundamental $\mathrm{SH}\left(\beta_{n}=\beta_{0}+2 n \pi / P\right)$, which provides for the worst case $\left(\theta_{\mathrm{RAD},-1}=-18^{\circ}, \beta_{0} / k_{0}=1.43\right)$ a value for $\beta_{-2} / k_{0}=-2.048$.

For fixed values of $W_{m}(y)$ and $M(y)$, the corresponding value of the complex wavenumber is now determined by applying the Bloch-Floquet theorem to extract the $A B C D$ param-

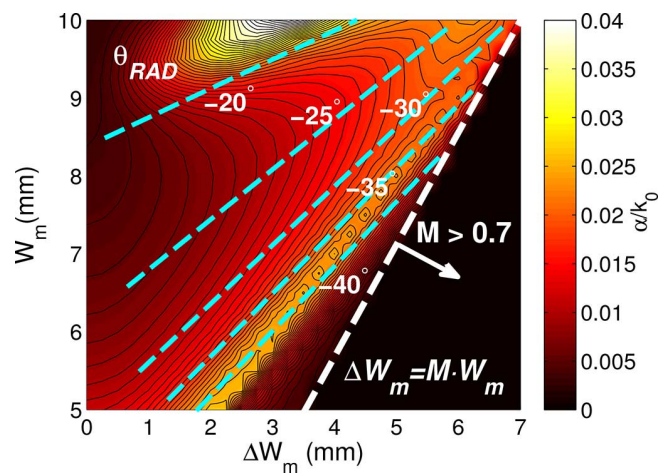

Fig. 3. Two-dimensional dispersion chart at the design frequency of $15 \mathrm{GHz}$ for the leakage rate $\alpha$ and the radiation angle $\theta_{\mathrm{RAD}}$ of the $n=-1$ space harmonic of the sinusoidally modulated HMLWA.

eters from a full-wave analysis of the HMLWA [34]. Varying $W_{m}(y)$ and $M(y)$ allows then for a control of the radiation angle and the leakage rate as illustrated in Fig. 3 with a two-dimensional dispersion chart for the $n=-1 \mathrm{SH}$. The values for $\theta_{\mathrm{RAD}}$ and $\alpha$ plotted in this figure correspond to a range of useful values of $W_{m}$ from $5 \mathrm{~mm}$ to $10 \mathrm{~mm}$, and their corresponding variations in width, $\Delta \mathrm{W}_{m}=W_{m} M$, from $0 \mathrm{~mm}$ to $7 \mathrm{~mm}$ [these variations in $\Delta \mathrm{W}_{m}=W_{m} M$ come determined by the oscillating part in (5)]. In particular, it is observed that the leakage rate $\alpha$ increases with $\Delta \mathrm{W}_{m}$ for a constant value of $W_{m}$, which can be explained by a higher excitation of the $n=-1 \mathrm{SH}$ due to a deeper modulation index $M$, as commonly occurs in SM LWAs [1], [19]-[21]. On the other hand, increasing values of $W_{m}$ provide higher radiation angles $\theta_{\mathrm{RAD}}$ tending to broadside $\theta=0^{\circ}$ (isocurves plotted with dashed blue line), eventually entering the open stop band zone for $W_{m}>9 \mathrm{~mm}$ and $\Delta W_{m}<4$ $\mathrm{mm}$. It can also be observed in Fig. 3 that this mutual influence of $W_{m}$ and $\Delta \mathrm{W}_{m}$ over both the phase constant and the leakage rate provides a nearly linear dependence with $\theta_{\mathrm{RAD}}$.

In order to show the capability to control both the amplitude and phase of the radiated fields for the $n=-1$ space harmonic, a tapered illumination function has been synthesized to obtain a focused near-field pattern at the design frequency of $15 \mathrm{GHz}$ (see Fig. 4). The focal height has been fixed at $z_{F}=5.5 \lambda_{0}=$ $110 \mathrm{~mm}$ and the longitudinal position of the focal point is set to $y_{F}=-0.65 \lambda_{0}=-13 \mathrm{~mm}$, which will allow that several 1-D LWAs can be arranged around a central point $(y=0)$ to obtain a 3-D near-field focus, as it will be shown in the next sections. This prescribed focal position requires the variation for $\theta_{\mathrm{RAD}}(y)$ and $\alpha(y) / k_{0}$ shown in Fig. 4(a), which provides a converging phase front over the focal position as described in [35], [36]. The corresponding theoretical near-field pattern obtained with an in-house code developed by some of the authors in [36] is plotted in Fig. 4(b), which shows that the fields have been suitably focused at the focal position $\left(z_{F}, y_{F}\right)$.

The variations of the tapered illumination function shown in Fig. 4(a) needs to be translated into their corresponding geometrical non-uniform sinusoidal modulation for $W(y)$. Thus, the data obtained in the dispersion chart of Fig. 3 are used as a look-up table in order to determine the requested values for $W_{m}(y)$ and $\Delta W_{m}(y)$, as shown in Fig. 5. The total value for 


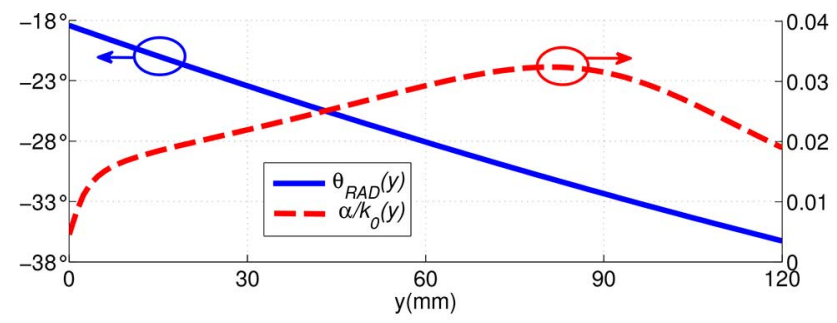

(a)

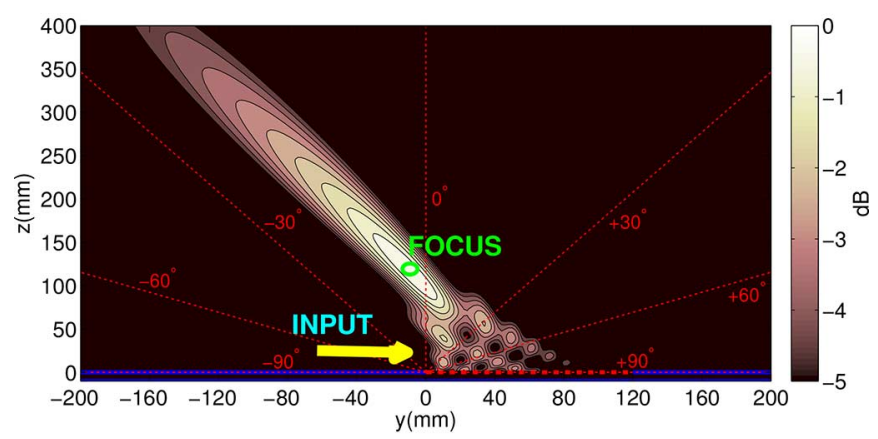

(b)

Fig. 4. Synthesis of a near-field focusing pattern at a focal distance $z_{F}=$ $5.5 \lambda_{0}=110 \mathrm{~mm}$ for a 1-D HMLWA with length $L_{A}=6 \lambda_{0}=120 \mathrm{~mm}$ at the design frequency of $15 \mathrm{GHz}$. (a) Tapering along the antenna length of $\theta_{\mathrm{RAD}}$ and $\alpha / k_{0}$. (b) Corresponding near-field pattern for the illustrated variation of the leaky mode.
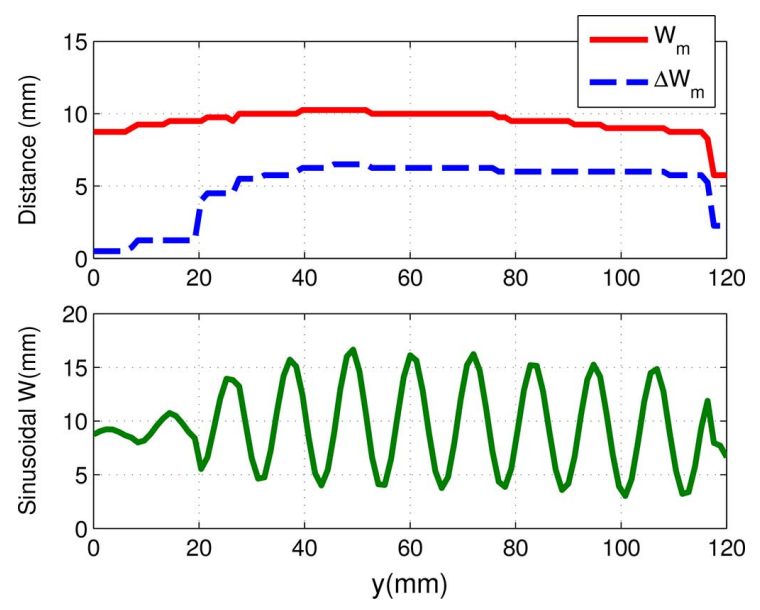

Fig. 5. Tapering along the antenna length of the width of the HMLWA to synthesize a near-field focusing pattern at the design frequency of $15 \mathrm{GHz}$.

the strip width $W$ along the complete radiating length is also plotted in Fig. 5.

\section{Design of the Radial ArRay Lens}

From the design of the 1-D sinusoidally modulated HMLWA described in Section II, which shows the capability to radiate backward and to provide 2-D near-field focusing, a radial array configuration has been conceived with the purpose of obtaining a 3-D near-field focus over the center of the array. Conventionally, microwave near-field focusing lenses have been based on the ability to focus the radiated electromagnetic fields within a prescribed region of the free space. Two main approaches have

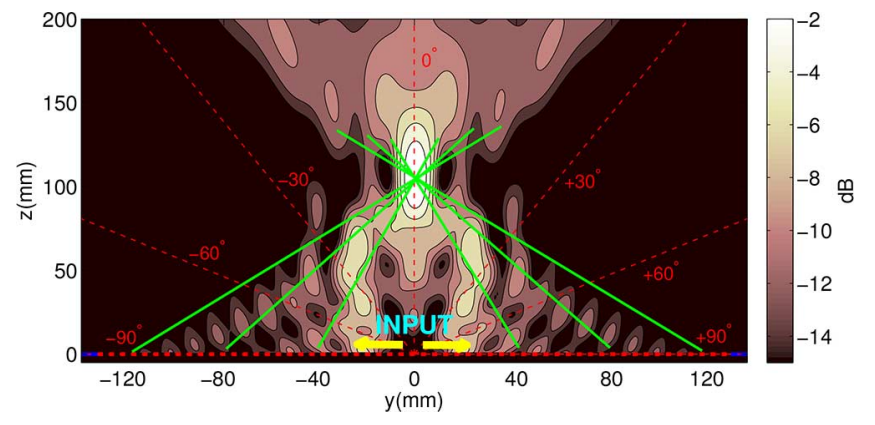

Fig. 6. Simulated near fields for two symmetrically fed HMLWAs with a tapered illumination function at the design frequency of $15 \mathrm{GHz}$.

been reported in the literature: i) to conform the radiating element so that all sections point to the same focal region as proposed in [37], and ii) to taper the radiated fields by changing the electrical properties along the device and thus avoiding to curve it [35]. Based on this second approach, there have been proposed several technologies and topologies such as focusing plates that require of an external source [38], focusing systems inside parallel-plate waveguides [29], or more integrated devices as in [39], [40]. Other possible designs have been conceived from tapered far-field radiations patterns that make use of a quadratic phase taper to approximate the radiated near-field pattern [41], or by using Bessel beams [42], [43].

This work follows the second approach but makes use of the structure proposed in Section II with capability to simultaneously and flexibly taper both the amplitude and phase of the radiated leaky-wave fields. Moreover, the leaky wave must radiate backward to keep a simple central feeding of the structure as in [39]-[41] and to obtain a single near-field focus over the central feeding point. As a first validation of the proposed focusing system, a simpler design based on two symmetric 1-D HMLWAs is first considered. Fig. 6 shows the simulated fields for two symmetrically fed 1-D HMLWAs that have been tapered along their lengths to produce the leaky-wave near fields shown in Fig. 4. It can be seen that the focusing pattern is obtained at the center position $(y=0)$ and at the prescribed focal height $z_{F}=5.5 \lambda_{0}=110 \mathrm{~mm}$. The variation of the local pointing angle along the antenna length has been marked with green lines to highlight the tapering of the local effective radiation angle $\theta_{\mathrm{RAD}}(y)$ of the two corresponding leaky waves propagating at opposite directions.

The symmetric configuration with two HMLWAs can only provide 2-D near-field focusing [29] in the $z y$-plane, in the form of a fan-focused beam in the $x z$-plane (as usually happens with 1-D line source LWAs). In order to obtain a 3-D focus it is proposed an innovative configuration formed by $N=81$-D HMLWAs radially arranged around a central fed. The proposed design is shown in Fig. 7 together with an illustrative view of the desired focal region and the different contributions of all sections of the antennas.

As each HMLWA is polarized linearly with its radiated $E$-field contained within the $x y$-plane [31], the polarization of the complete array is defined as the result of combining all the individual polarizations of each 1-D HMLWA. This resulting polarization is illustrated in Fig. 8, where the radiated 


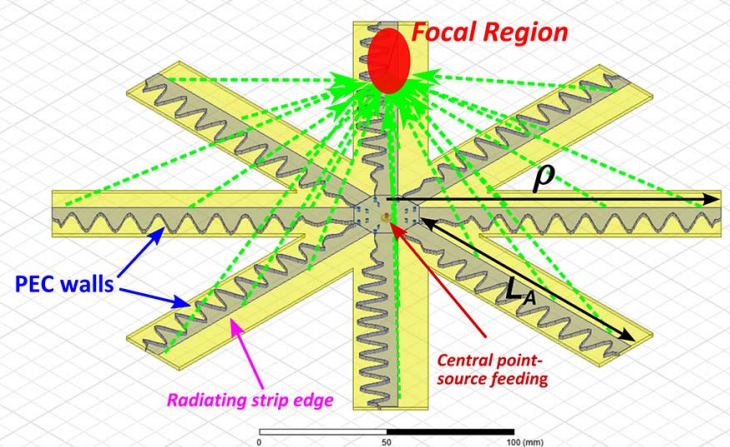

Fig. 7. Scheme of the 3-D near-field focusing system consisting of a radial array with 8 sinusoidally modulated HMLWAs.

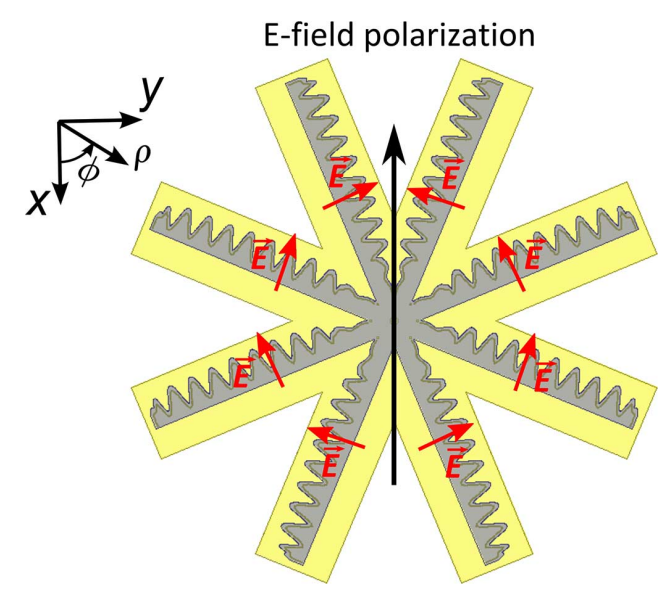

Fig. 8. $E$-field polarization arrangement for each element of the array (red arrows), and resulting array polarization (black arrow).

$E$-field for each HMLWA is represented with a red arrow and the resulting array polarization at the focal point is represented with a black arrow.

To confirm the desired near-field focusing behavior of the designed radial array, a full-wave simulation (HFSS [44]) of the near fields in linear scale is plotted in Fig. 9 at the design frequency of $15 \mathrm{GHz}$ for the plane $\phi=45^{\circ}$. This simulation shows that the desired near-field focusing pattern can actually be obtained by the arrangement of the $N=8$ HMLWAs.

Moreover, in order to support the theoretical approach given in Fig. 8 on the polarization of the complete array, results for the simulated electric field vector are shown in Fig. 10 at a focal distance $z_{F}=5.5 \lambda_{0}=110 \mathrm{~mm}$. In particular, Fig. 10(a) shows an electric field oriented along $-x$ axis for a time phase $=$ $0^{\circ}$ whereas in Fig. 10(b) the vectors are oriented in opposite direction along $+x$ axis for a time phase $=180^{\circ}$. It can also be observed that the maximum amplitude of the radiated field is located at the center of the array (shown by the red color vectors for both time phases) as a result of the focusing pattern.

An important characteristic of the proposed radial array is that it can simply be fed by a single coaxial probe. A detailed view of the employed feeding is shown in Fig. 11. Specifically, the inner conductor of the coaxial (highlighted in pink color) passes through the substrate and then is soldered to the metal top layer (as can also be seen in Fig. 12). Moreover, it can be observed that the 1-D HMLWAs are radially arranged around a radius $R_{0}$

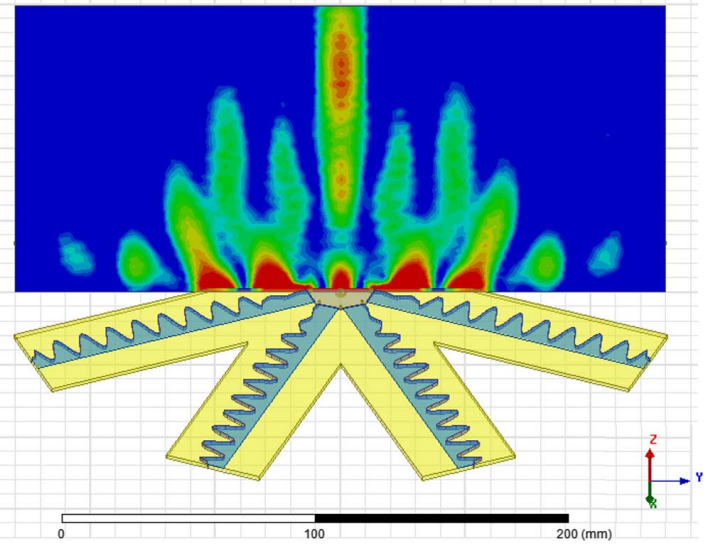

Fig. 9. Simulated near-fields for the designed radial array of 8 sinusoidally modulated HMLWAs at the design frequency of $15 \mathrm{GHz}$.
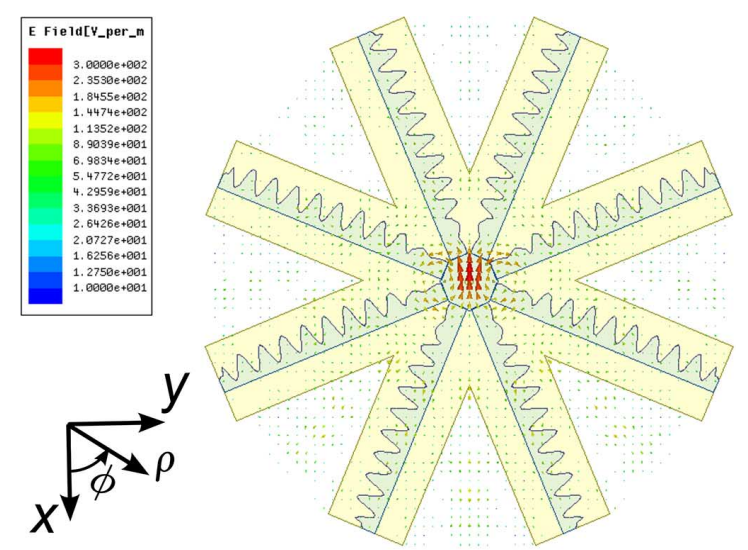

(a)
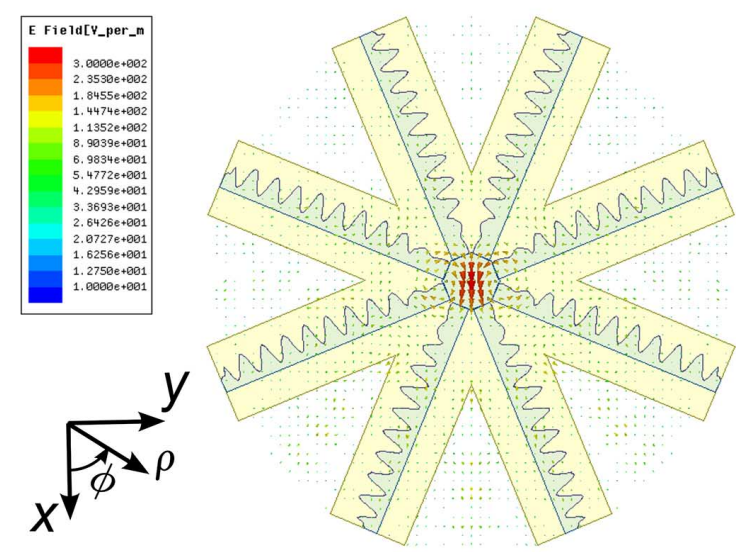

(b)

Fig. 10. Simulated electric field vector, $E(x, y, z)(\mathrm{V} \backslash \mathrm{m})$, at a focal distance $z_{F}=5.5 \lambda_{0}=110 \mathrm{~mm}$ : (a) time phase $=0^{\circ}$ and (b) time phase $=180^{\circ}$.

from the coaxial probe. As seen in Fig. 11, the PEC wall of the HMLWAs is approximated by means of a row of closely spaced metallic via holes that are separated a distance $s=1 \mathrm{~mm}$ and have a diameter $d=0.5 \mathrm{~mm}$ for the modulated HMLWA part. The vias located at the beginning of each HMLWA have a diameter of $d_{0}=1 \mathrm{~mm}$ in order to improve the input matching. Each antenna has been terminated in a short circuit also formed by metallic vias holes located at the end part of the HMLWAs 


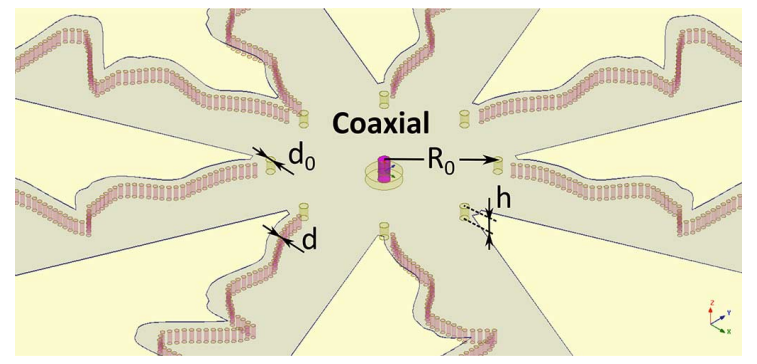

Fig. 11. Detailed view of the employed coaxial feeding with some of its main geometrical parameters: $R_{0}=13 \mathrm{~mm}, d=0.5 \mathrm{~mm}, d_{0}=1 \mathrm{~mm}$ and $h=$ $1.57 \mathrm{~mm}$.

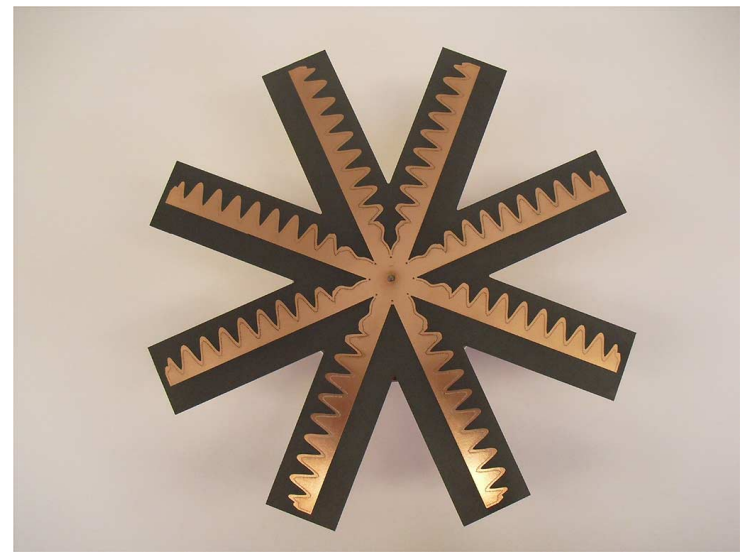

Fig. 12. Top photographic view of the manufactured prototype of the radial array with $N=8$ sinusoidally modulated HMLWAs.

(see Fig. 12). However, this termination has negligible influence on the array focusing pattern since only a small part of the energy reaches the far end of the antenna due to the high designed radiation efficiency $\left(\eta_{\mathrm{RAD}} \approx 90 \%\right)$.

\section{MeAsured Results}

In this section measured results of the near-field focusing lens are shown for the design frequency of $15 \mathrm{GHz}$. Several figures of merit such as focal depth, focal width, and input matching have been obtained. The designed radial array has been manufactured using the commercial substrate Taconic TLY-5 with thickness $h=1.57 \mathrm{~mm}$, relative permittivity $\epsilon_{r}=2.2$, and loss tangent $\tan \delta=0.0009$. A photograph of the prototype is shown in Fig. 12 , where eight HMLWAs radially arranged around a central feeding can be observed.

As explained in previous sections, the proposed radial array configuration possesses the capability to focus the radiated near fields into a prescribed 3-D focal region above the center of the array, acting as the microwave lens schemed in Fig. 7. To experimentally validate this concept, the radial array has been measured by means of a near-field measurement system equipped with a standard rectangular waveguide WR62 as measurement probe. Fig. 13 shows a photograph of the setup employed to measure the radiated fields along a $x y$-plane for an arbitrary distance $z$.

In order to obtain the real performance of the proposed structure and thus its focusing capability, several cuts at the focal height $z=5.5 \lambda_{0}=110 \mathrm{~mm}$ for different azimuthal angles

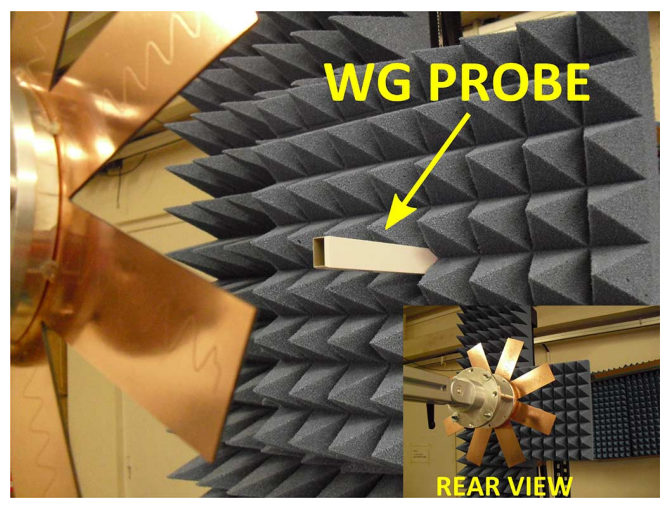

Fig. 13. Photographs of the near-field measurement setup used for the radial array.

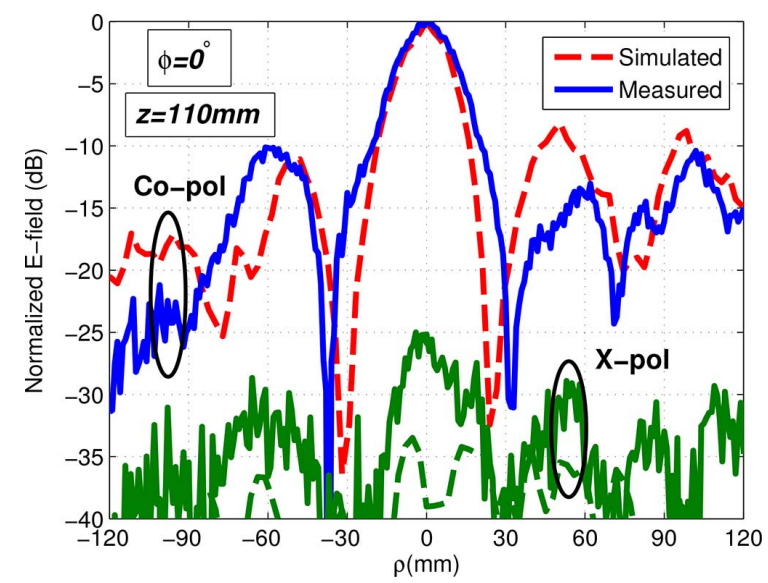

Fig. 14. Measured and simulated transverse cuts along $\rho$ for the focal height $z_{F}=110 \mathrm{~mm}$ at the plane $\phi=0^{\circ}$.

$\phi$ and polarizations (Co-pol and X-pol) have been measured. Specifically, the Co-pol component is defined along the main polarization of the array $E_{x}$ and the X-pol component along the orthogonal polarization $E_{y}$ (see Fig. 8). Fig. 14 shows the normalized radiated $E$-field along a transverse plane at $\phi=$ $0^{\circ}$ and a focal height $z=5.5 \lambda_{0}=110 \mathrm{~mm}$. Measured results (continuous line) are compared with HFSS simulations (dashed line) obtaining acceptable agreement. Particularly, for the Co-pol component the focal width $\Delta \rho$ is correctly estimated, which at $-3 \mathrm{~dB}$ is $\Delta \rho=20 \mathrm{~mm}$. However, it is observed a slight increment of the side-lobe levels around the focus, which can be explained by the perturbation introduced by the measurement probe. On the other hand, the X-pol component, which is represented in green color, is $25 \mathrm{~dB}$ below the Co-pol component, showing no significant influence at this cut.

The transverse cut for the $E$-field along $\phi=45^{\circ}$ at the prescribed focal height of $z=5.5 \lambda_{0}=110 \mathrm{~mm}$ is shown in Fig. 15. Measured results are compared with simulations and the agreement for this cut is also good, showing for the Co-pol component a focal width at $-3 \mathrm{~dB}$ of $\Delta \rho=18 \mathrm{~mm}$, similar to the one obtained at $\phi=0^{\circ}$ (the small difference can be attributed to the lack of symmetry along $\phi$ as a result of the necessary flip of the SM HMLWA to obtain linear polarization as previously explained with reference to Fig. 8). This lack of symmetry is also observed in the X-pol component, which is more than $5 \mathrm{~dB}$ 


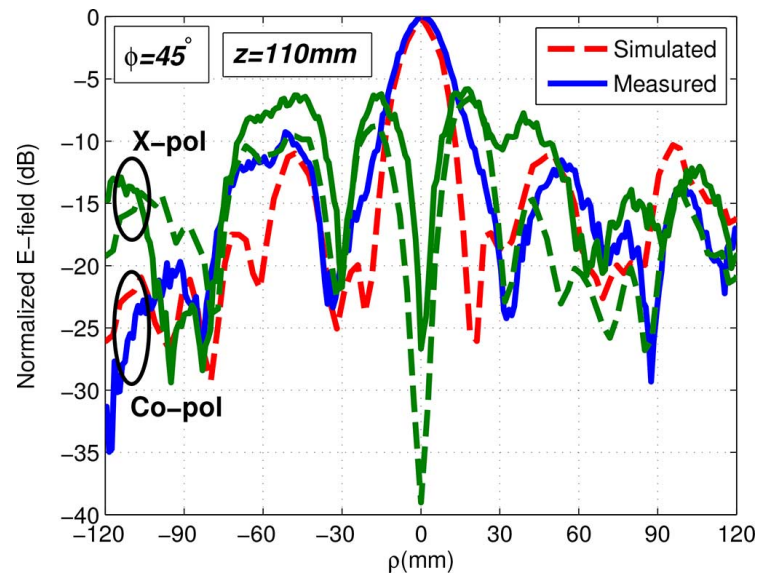

Fig. 15. Measured and simulated transverse cuts along $\rho$ for the focal height $z_{F}=110 \mathrm{~mm}$ at the plane $\phi=45^{\circ}$.

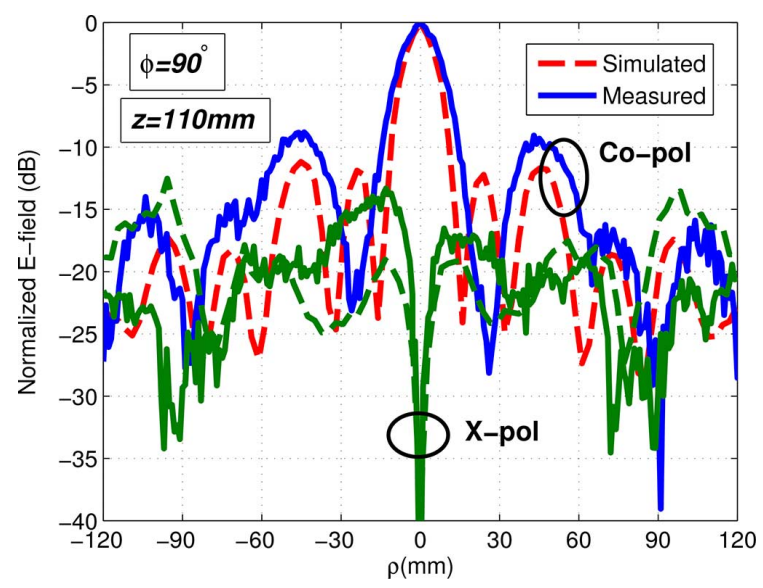

Fig. 16. Measured and simulated transverse cuts along $\rho$ for the focal height $z_{F}=110 \mathrm{~mm}$ at the plane $\phi=90^{\circ}$.

below the Co-pol component and shows a radiation null at the central position.

A transverse cut along $\phi=90^{\circ}$ is represented in Fig. 16 at the focal height $z=5.5 \lambda_{0}=110 \mathrm{~mm}$. As in the previous cuts, the correspondence between simulations and measurements is good. For this cut, the focal width for the Co-pol component at $-3 \mathrm{~dB}$ is $\Delta \rho=15 \mathrm{~mm}$, comparable to the values obtained for other angles. Moreover, it is observed that the X-pol component is $15 \mathrm{~dB}$ below the Co-pol component and shows a radiation null at the central position as in the cut for $\phi=45^{\circ}$. However, it should also be noted that due to polarization mismatches between the resulting array polarization and the polarization of each element (see Fig. 8), as well as the use of a tapered illumination function, the obtained focal width for the Co-pol component $(\Delta \rho)$ is larger than the one achievable for the ideal case of a uniformly illuminated circular aperture [45], [46] (which would provide an approximate value of $\Delta \rho \approx 0.42 \lambda_{0}$ according to $\Delta \rho \approx \lambda_{0} F / D$, with $F$ being the focal length and $D$ the diameter of the aperture).

Another important parameter to evaluate the performance of the proposed device as a microwave lens is the focal depth $\Delta z$; i.e., the half-power width of the focus along the $z$-axis. Fig. 17 shows an axial cut for the normalized Co-pol compo-

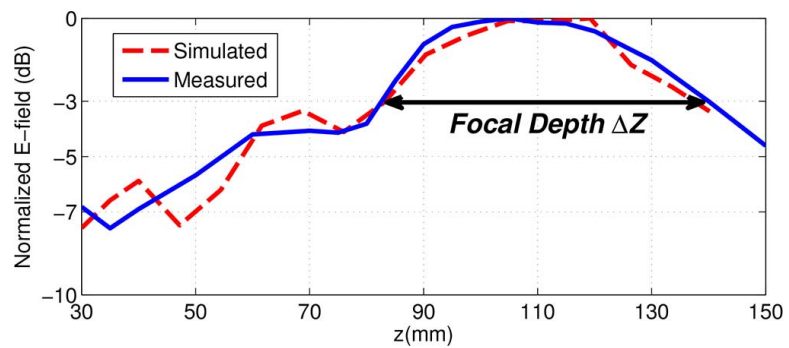

Fig. 17. Measured and simulated axial cuts along $z$-axis at the center position $\rho=0$.

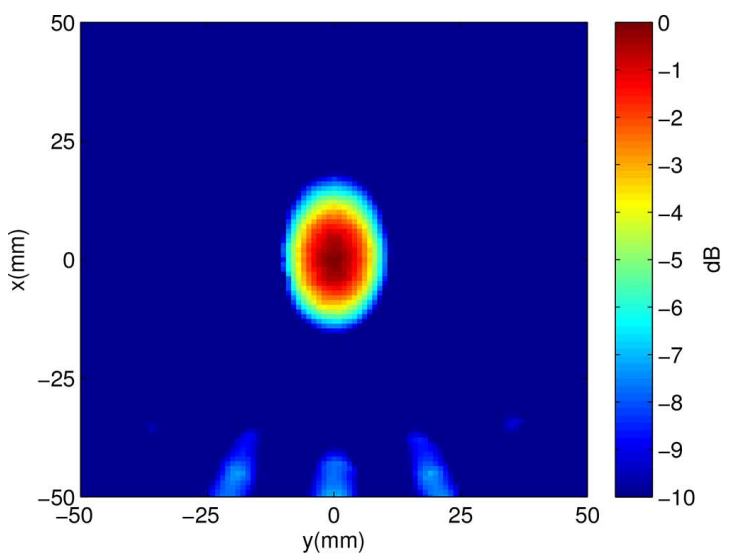

(a)

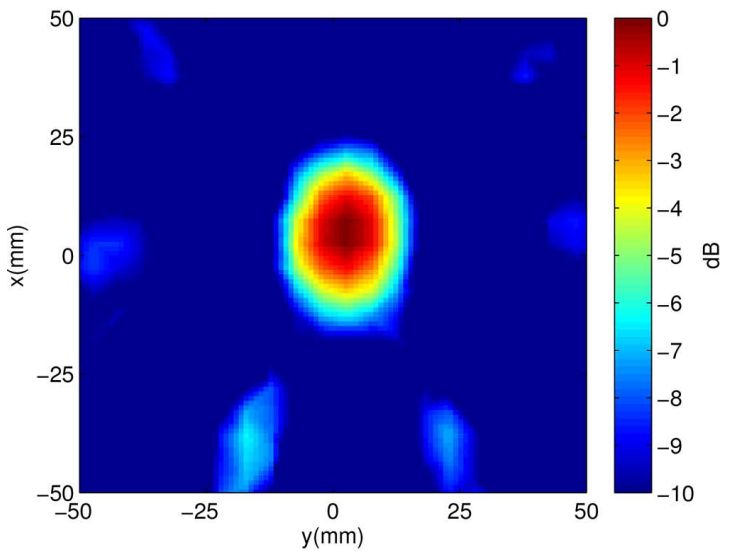

(b)

Fig. 18. Near-field plane at the focal height $z_{F}=110 \mathrm{~mm}$ for the design frequency of $15 \mathrm{GHz}$ : (a) simulated and (b) measured.

nent of the $E$-field along the $z$-axis at the central position of $\rho=0$. The comparison of measured results with simulations shows good correspondence between them. A focal depth of $\Delta z$ $=60 \mathrm{~mm}$ is obtained, approximately three times larger than the focal width. This ellipsoidal shape of the focal region (prolate spheroid) is common to all planar near-field focused lenses [28], [29], [38]-[41] as a result of their 2-D (planar) aperture topology. The capability to focus the energy also along the axial direction demonstrates that a 3-D focus is actually obtained at the prescribed focal region of $\rho=0$ and $z=5.5 \lambda_{0}=110 \mathrm{~mm}$ for the design frequency of $15 \mathrm{GHz}$.

Transverse cuts in the $x y$-plane for the focal distance $z=$ $z_{F}=110 \mathrm{~mm}$ are represented in Fig. 18 for the Co-pol component, where measured near fields for the $x y$-plane at the focal 


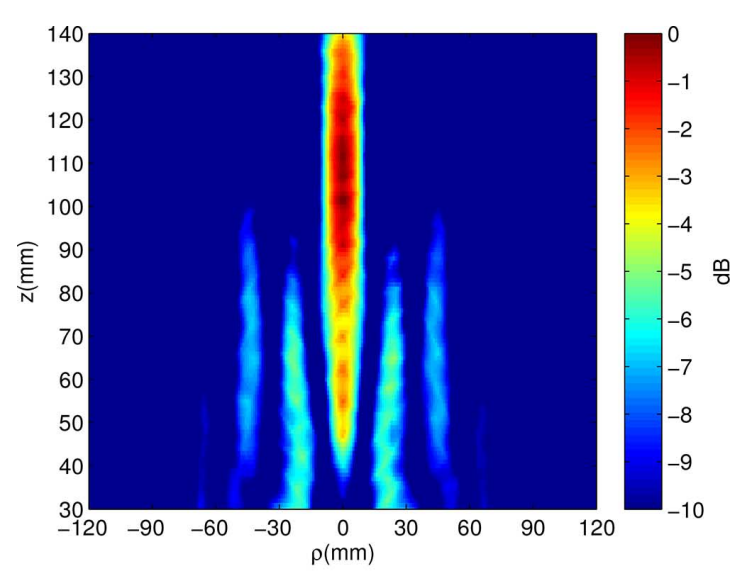

(a)

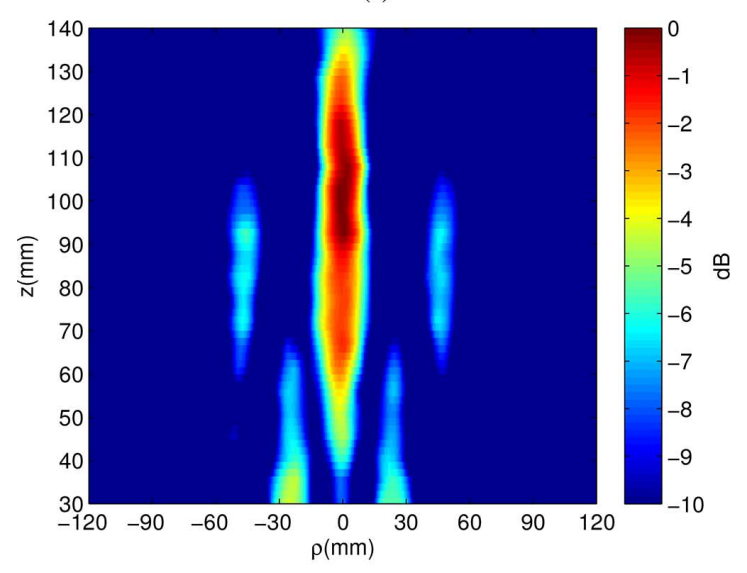

(b)

Fig. 19. Near-field plane at $\phi=0^{\circ}$ along the $z$-axis for the design frequency of $15 \mathrm{GHz}$ : (a) simulated and (b) measured.

$z=5.5 \lambda_{0}=110 \mathrm{~mm}$ are compared with simulated ones. A clearly symmetric (circular) focused spot in the $x y$-plane is obtained in congruence with the plotted values of the different transverse and axial cuts. Moreover, it can be seen that the measured focusing pattern shown in Fig. 18(b) is slightly wider than the simulated one shown in Fig. 18(a). These differences can be attributed to small discrepancies between the structure simulated in HFSS and the measured prototype as well as by the perturbation introduced by the measurement probe.

Fig. 19 shows the simulated and measured near-fields (Copol) for the $\rho z$-plane at $\phi=0^{\circ}$. It can be observed that the highest intensity (plotted in red color) is obtained at the focal height $z_{F}=110 \mathrm{~mm}$. Both results show a similar focusing pattern, demonstrating a good agreement and that a 3-D nearfield focus above the center of the array has been achieved.

Finally, the measured $S_{11}$ parameter is compared with simulations in Fig. 20. Although some discrepancies are observed between both results, as a result of manufacturing problems with the gap left for the inner conductor of the coaxial probe, the key point is that a good input matching $\left(S_{11}<-15 \mathrm{~dB}\right)$ is obtained at the design frequency of $15 \mathrm{GHz}$ and over a band of $\sim 0.5$ $\mathrm{GHz}$. It should also be highlighted that this type of leaky-wave lenses are strongly dispersive with frequency as demonstrated in [36], [40]. As a consequence, the designed focal length, $F$, is only kept for the design frequency, increasing its position

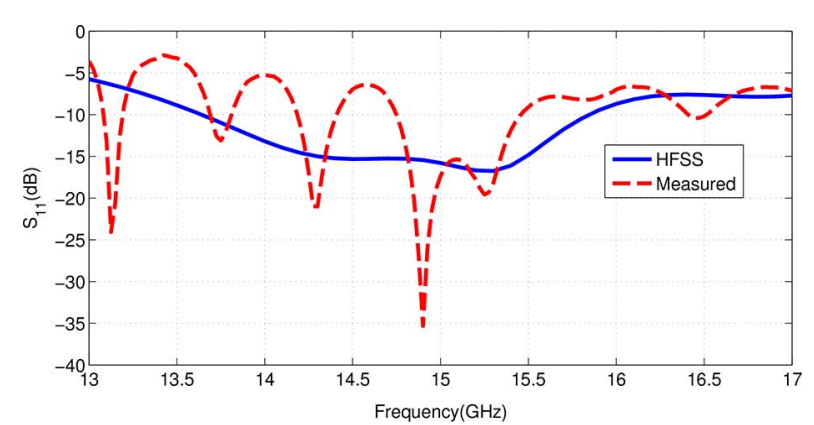

Fig. 20. Measured and simulated input matching for the radial array of $N=8$ sinusoidally modulated HMLWAs.

for higher frequencies until it eventually breaks into a conical shaped focused spot, as shown in [40].

\section{CONCLUSION}

An innovative type of microwave lens conceived from an array of sinusoidally modulated half-mode leaky-wave antennas (HMLWAs) has been reported. The array consists of eight HMLWAs radially arranged around a single central feeding. The HMLWAs have been sinusoidally modulated in order to excite Floquet space harmonics that allow for backward radiation. Each HMLWA has been designed only to radiate with the $n=-1$ space harmonic. In particular, by modifying the modulation index of the sinusoidal function and the width of the HMLWA, a simultaneous control of both the amplitude and phase of the radiated fields has been obtained, which has made it possible the design of a shaped near-field focusing pattern. This fact in combination with the radial arrangement of the HMLWAs has allowed us to obtain a 3-D focus above the center of the array. The proposed design of the microwave lens has been validated with near-field measurements for the design frequency of $15 \mathrm{GHz}$. The present novel design demonstrates the utility of non-uniform sinusoidally modulated leaky-wave antennas in order to freely synthesize complex aperture distributions (in both amplitude and phase).

\section{REFERENCES}

[1] A. Oliner and A. Hessel, "Guided waves on sinusoidally-modulated reactance surfaces," IRE Trans. Antennas Propag., vol. 7, no. 5, pp. 201-208, Dec. 1959.

[2] T. Tamir, H. C. Wang, and A. Oliner, "Wave propagation in sinusoidally stratified dielectric media," IEEE Trans. Microw. Theory Tech., vol. 12, no. 3, pp. 323-335, May 1964.

[3] A. H. Nayfeh and O. Asfar, "Parallel-plate waveguide with sinusoidally perturbed boundaries," J. Appl. Phys., vol. 45, no. 11, pp. 4797-4800, Nov. 1974.

[4] A. K. Mallick and G. Sanyal, "Electromagnetic wave propagation in a rectangular waveguide with sinusoidally varying width," IEEE Trans. Microw. Theory Tech., vol. 26, no. 4, pp. 243-249, Apr. 1978.

[5] A. W. Snyder and R. A. Sammut, "Radiation from optical waveguides: Leaky-mode interpretation," Electron. Lett., vol. 15, no. 2, pp. 58-60, Jan. 1979.

[6] G. Kowalski, "Microstrip meanderlines," AEU-Int. J. Electron. Commun., vol. 29, no. 6, pp. 248-250, 1975.

[7] N. V. Nair and K. Mallick, "An analysis of a width-modulated microstrip periodic structure," IEEE Trans. Microw. Theory Tech., vol. 32, no. 2, pp. 200-204, Feb. 1984.

[8] C. Caloz, "Metamaterial dispersion engineering concepts and applications," Proc. IEEE, vol. 99, no. 10, pp. 1711-1719, Oct. 2011.

[9] C. Holloway, E. F. Kuester, J. Gordon, J. O'Hara, J. Booth, and D. Smith, "An overview of the theory and applications of metasurfaces: The two-dimensional equivalents of metamaterials," IEEE Antennas Propag. Mag., vol. 54, no. 2, pp. 10-35, Apr. 2012. 
[10] S. Maci, G. Minatti, M. Casaletti, and M. Bosiljevac, "Metasurfing: Addressing waves on impenetrable metasurfaces," IEEE Antennas Wireless Propag. Lett., vol. 10, pp. 1499-1502, 2011.

[11] T. Lopetegi, M. A. G. Laso, J. Hernandez, M. Bacaicoa, D. Benito, M. Garde, M. Sorolla, and M. Guglielmi, "New microstrip "wigglyline" filters with spurious passband suppression," IEEE Trans. Microw. Theory Tech., vol. 49, no. 9, pp. 1593-1598, Sept. 2001.

[12] L. Matekovits, G. C. V. Colomé, and M. Orefice, "Controlling the bandlimits of TE-surface wave propagation along a modulated microstripline-based high impedance surface," IEEE Trans. Antennas Propag., vol. 56, no. 8, pp. 2555-2562, Aug. 2008.

[13] M. A. G. Laso, T. Lopetegi, M. J. Erro, D. Benito, M. Garde, M. Muriel, M. Sorolla, and M. Guglielmi, "Real-time spectrum analysis in microstrip technology," IEEE Trans. Microw. Theory Tech., vol. 51, no. 3, pp. 705-717, Mar. 2003.

[14] M. Nannetti, F. Caminita, and S. Maci, "Leaky-wave based interpretation of the radiation from holographic surfaces," in Proc. IEEE Antennas and Propagation Soc. Int. Symp., Honolulu, HI, USA, Jun. 2007, pp. 5813-5816.

[15] S. Podilchak, A. Freundorfer, and Y. Antar, "Planar leaky-wave antenna designs offering conical-sector beam scanning and broadside radiation using surface-wave launchers," IEEE Antennas Wireless Propag. Lett., vol. 7, pp. 155-158, 2008.

[16] A. Sutinjo, M. Okoniewski, and R. Johnston, "A holographic antenna approach for surface wave control in microstrip antenna applications," IEEE Trans. Antennas Propag., vol. 58, no. 3, pp. 675-682, Mar. 2010.

[17] B. Fong, J. Colburn, J. Ottusch, J. Visher, and D. Sievenpiper, "Scalar and tensor holographic artificial impedance surfaces," IEEE Trans. Antennas Propag., vol. 58, no. 10, pp. 3212-3221, Oct. 2010.

[18] G. Minatti, F. Caminita, M. Casaletti, and S. Maci, "Spiral leaky-wave antennas based on modulated surface impedance," IEEE Trans. Antennas Propag., vol. 59, no. 12, pp. 4436-4444, Dec. 2011.

[19] Z. Mekkioui, "Determination of non uniform sinusoidal microstrip leaky-wave antenna radiating performances in millimeter band," Int. J. Elect. Electron. Eng., vol. 5, no. 3, pp. 151-155, 2011.

[20] A. Patel and A. Grbic, "A printed leaky-wave antenna based on a sinusoidally-modulated reactance surface," IEEE Trans. Antennas Propag., vol. 59, no. 6, pp. 2087-2096, Jun. 2011

[21] S. Podilchak, L. Matekovits, A. Freundorfer, Y. Antar, and M. Orefice, "Controlled leaky-wave radiation from a planar configuration of widthmodulated microstrip lines," IEEE Trans. Antennas Propag., vol. 61 , no. 10 , pp. 4957-4972, Oct. 2013.

[22] L. Matekovits, "Analytically expressed dispersion diagram of unit cells for a novel type of holographic surface," IEEE Antennas Wireless Propag. Lett., vol. 9, pp. 1251-1254, 2010.

[23] L. Matekovits and Y. Ranga, "Controlling the phase of the scattered and/or radiated field from a high impedance surface of quasi-periodic sequences," IEEE Antennas Wireless Propag. Lett., vol. 12, pp. 321-324, 2013.

[24] S. Lim, C. Caloz, and T. Itoh, "Metamaterial-based electronically controlled transmission-line structure as a novel leaky-wave antenna with tunable radiation angle and beamwidth," IEEE Trans. Microw. Theory Tech., vol. 53, no. 1, pp. 161-173, Jan. 2005

[25] Q.-H. Lai, W. Hong, Z.-Q. Kuai, Y. S. Zhang, and K. Wu, "Halfmode substrate integrated waveguide transverse slot array antennas," IEEE Trans. Antennas Propag., vol. 57, no. 4, pp. 1064-1072, Apr. 2009.

[26] Y. Li, Q. Xue, E.-N. Yung, and Y. Long, "The periodic half-width microstrip leaky-wave antenna with a backward to forward scanning capability," IEEE Trans. Antennas Propag., vol. 58, no. 3, pp. 963-966, Mar. 2010.

[27] Q. Lai, C. Fumeaux, and W. Hong, "Periodic leaky-wave antennas fed by a modified half-mode substrate integrated waveguide," IET Microw. Antennas Propag., vol. 6, no. 5, pp. 594-601, Apr. 2012.

[28] A. J. Martinez-Ros and J. L. Gomez-Tornero, "Free space near field focusing from an array of sinusoidally modulated half-mode LWAs," in Proc. IEEE Antennas and Propagation Soc. Int. Symp. (APSURSI), Orlando, FL, USA, Jul. 2013, pp. 1568-1569.

[29] A. J. Martinez-Ros, J. L. Gomez-Tornero, F. J. Clemente-Fernandez, and J. Monzo-Cabrera, "Microwave near-field focusing properties of width-tapered microstrip leaky-wave antenna," IEEE Trans. Antennas Propag., vol. 61, no. 6, pp. 2981-2990, Jun. 2013.

[30] A. A. Oliner, "Leakage from higher modes on microstrip line with application to antennas," Radio Sci., vol. 22, pp. 907-912, Nov. 1987.

[31] G. Zelinski, G. Thiele, M. Hastriter, M. Havrilla, and A. Terzuoli, "Half width leaky wave antennas," IET Microw. Antennas Propag., vol. 1, no. 2, pp. 341-348, Apr. 2007.
[32] A. A. Oliner and D. R. Jackson, , J. L. Volakis, Ed., "Leaky-wave antennas," in Antenna Engineering Handbook, 4th ed. New York, NY USA: McGraw-Hill, Jun. 2007, ch. 11

[33] A. J. Martinez-Ros, J. L. Gomez-Tornero, and G. Goussetis, "Holographic pattern synthesis with modulated substrate integrated waveguide line-source leaky-wave antennas," IEEE Trans. Antennas Propag., vol. 61, no. 7, pp. 3466-3474, 2013.

[34] D. M. Pozar, Microwave Engineering, 2nd ed. New York, NY, USA Wiley, 1998

[35] P. Burghignoli, F. Frezza, A. Galli, and G. Schettini, "Synthesis of broad-beam patterns through leaky-wave antennas with rectilinear geometry," IEEE Antennas Wireless Propag. Lett., vol. 2, no. 1, pp. $136-139,2003$.

[36] J. L. Gomez-Tornero, F. Quesada-Pereira, A. Alvarez-Melcon, G. Goussetis, A. Weily, and Y. Guo, "Frequency steerable two dimensional focusing using rectilinear leaky-wave lenses," IEEE Trans. Antennas Propag., vol. 59, no. 2, pp. 407-415, Feb. 2011.

[37] I. Ohtera, "Diverging/focusing of electromagnetic waves by utilizing the curved leakywave structure: Application to broad-beam antenna for radiating within specified wide-angle," IEEE Trans. Antennas Propag. vol. 47, no. 9, pp. 1470-1475, Sep. 1999.

[38] A. Grbic and R. Merlin, "Near-field focusing plates and their design," IEEE Trans. Antennas Propag., vol. 56, no. 10, pp. 3159-3165, Oct. 2008.

[39] J. L. Gomez-Tornero, D. Blanco, E. Rajo-Iglesias, and N. Llombart, "Holographic surface leaky-wave lenses with circularly-polarized focused near-fields. Part I: Concept, design and analysis theory," IEEE Trans. Antennas Propag., vol. 61, no. 7, pp. 3475-3485, Jul. 2013.

[40] D. Blanco, J. L. Gomez-Tornero, E. Rajo-Iglesias, and N. Llombart, "Holographic surface leaky-wave lenses with circularly-polarized focused near-fields. Part II: Experiments and description of frequency steering of focal length," IEEE Trans. Antennas Propag., vol. 61, no. 7, pp. 3486-3494, Jul. 2013.

[41] M. Ettorre, M. Casaletti, G. Valerio, R. Sauleau, L. Le Coq, S. Pavone, and M. Albani, "On the near-field shaping and focusing capability of a radial line slot array," IEEE Trans. Antennas Propag., vol. 62, no. 4, pp. 1991-1999, Apr. 2014

[42] M. Ettorre and A. Grbic, "Generation of propagating Bessel beams using leaky-wave modes," IEEE Trans. Antennas Propag., vol. 60, no. 8, pp. 3605-3613, Aug. 2012

[43] P. Lemaitre-Auger, S. Abielmona, and C. Caloz, "Generation of Bessel beams by two-dimensional antenna arrays using sub-sampled distributions," IEEE Trans. Antennas Propag., vol. 61, no. 4, pp. 1838-1849, Apr. 2013.

[44] Ansoft HFSS v11 [Online]. Available: http://www.ansoft.com/products/hf/hfss/ 2007

[45] J. W. Sherman, III, "Properties of focused apertures in the Fresnel region," IRE Trans. Antennas Propag., vol. 10, no. 4, pp. 399-408, Jul. 1962.

[46] R. Hansen, "Focal region characteristics of focused array antennas," IEEE Trans. Antennas Propag., vol. 33, no. 12, pp. 1328-1337, Dec. 1985.

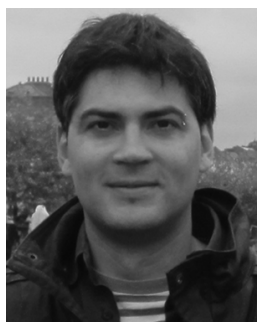

Alejandro Javier Martínez-Ros (S'11) was born in Cartagena, Spain, in 1981. He received the Telecommunication Engineer and Ph.D. degrees from the Universidad Politécnica de Cartagena (UPCT), Cartagena, Spain, in 2005 and 2014, respectively.

During 2006, he worked as an RF Engineer at the IMST GmbH in Kamp-Lintfort, Germany, where he was involved with the development of EBG structures for high precision antennas for the Galileo system, and with phase array and patch antennas for global navigation satellite systems. In 2007, he moved to Spain and worked as a Research Engineer in a local naval company, where he participated in the design of submarine telecommunication systems based on underwater acoustics. In 2008, he joined the Telecommunication and Electromagnetic Group (GEAT), UPCT, as a Research Assistant. He has been a visiting Ph.D. student at the Queen's University Belfast, Northern Ireland, U.K. His scientific research is mainly focused on the analysis and synthesis of leaky-wave devices for microwave and millimeter waveband applications.

Dr. Martínez-Ros was awarded a travel grant to attend the 2011 IEEE MTT-S IMWS in Sitges, Spain, the second prize for the best student paper at the 2012 Spanish URSI Symposium in Elche, Spain, and was a finalist for the best student paper at AP-S/URSI 2013 in Orlando, USA 


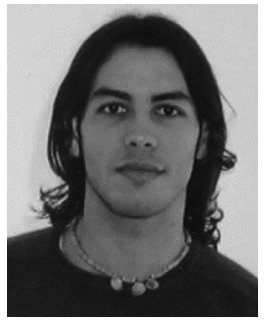

José Luis Gómez Tornero (M’06) was born in Murcia, Spain, in 1977. He received the Telecommunications Engineer degree from the Polytechnic University of Valencia (UPV), Valencia, Spain, in 2001, and the "laurea cum laude" Ph.D. degree in telecommunication engineering from the Technical University of Cartagena (UPCT), Cartagena, Spain, in 2005 .

In 1999 he joined the Radio Communications Department, UPV, as a Research Student, where he was involved in the development of analytical and numerical tools for the automated design of microwave filters in waveguide technology for space applications. In 2000, he joined the Radio Frequency Division, Industry Alcatel Espacio, Madrid, Spain, where he was involved with the development of microwave active circuits for telemetry, tracking and control (TTC) transponders for space applications. In 2001, he joined the Technical University of Cartagena (UPCT), Spain, as an Assistant Professor. From October 2005 to February 2009, he held the position of Vice Dean for Students and Lectures affairs in the Telecommunication Engineering Faculty at the UPCT. Since 2008, he has been an Associate Professor at the Department of Communication and Information Technologies, UPCT. His current research interests include: 1 . The development of numerical methods for the analysis and design of leaky-wave devices in planar and waveguide technologies; 2 . Their application for telecoms, RFID, microwave heating/sensing, wireless power transmission/harvesting, hyperthermia, and analog signal processing; 3 . The innovation in the area of higher-education teaching/learning. He has authored or coauthored over 50 peer-reviewed journal papers. He has been Visiting Researcher/Professor at the University of Loughborough, (U.K., England), Heriot-Watt University (U.K., Scotland), Queen's University of Belfast (U.K., Northern Ireland), and CSIRO-ICT Centre, Sydney, Australia.

Dr. Gómez Tornero received the 2004 National Award from the foundation EPSON-Ibérica to the best Ph.D. project in the field of Technology of Information and Communications (TIC). In June 2006, he received the Vodafone Foundation-COIT/AEIT (Colegio Oficial de Ingenieros de Telecomunicación) award to the best Spanish Ph.D. thesis in the area of Advanced Mobile Communications Technologies. This thesis was also awarded as the best thesis in the area of Electrical Engineering, by the Technical University of Cartagena in December 2006. In February 2010, he was appointed CSIRO Distinguished Visiting Scientist by the CSIRO ICT Centre, Sydney. He is the recipient or co-recipient of the 2010 IEEE Engineering Education conference award, the 2011 EuCAP best student paper prize, the 2012 EuCAP best antenna theory paper prize, and the 2012 and 2013 Spanish URSI prizes to the best student paper.

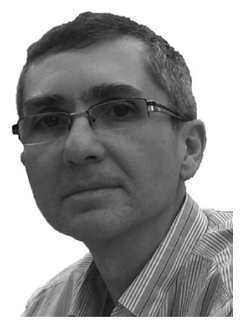

Vicente Losada was born in Sao Paulo (Brazil) in February 1969. He received the Licenciado and Ph.D. degrees from the University of Sevilla, Seville, Spain, in 1992 and 1997, respectively, both in physics.

He has been an Associate Professor in the Department of Applied Physics I, University of Seville, since 2004. His research interest includes manufacture and near field and far-field measurements techniques for antennas.

Prof. Losada acts as reviewer for the IEEE TRANSACtions on ANTENNAS AND PROPAGation.

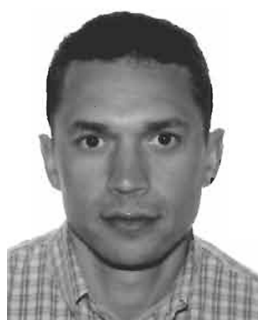

Francisco Mesa (M'93-SM'11-F'13) was born in Cádiz, Spain, in April 1965. He received the Licenciado and Doctor degrees in physics from the Universidad de Sevilla, Seville, Spain, in 1989 and 1991, respectively.

He is currently a Professor in the Departamento de Física Aplicada 1, Universidad de Sevilla, Seville, Spain. His research interests focus on electromagnetic propagation/radiation in planar structures.

Prof. Mesa has been serving as an Associate Editor of the IEEE TRANSACTIONS ON MICROWAVES, THEORY AND TECHNIQUES since 2013.

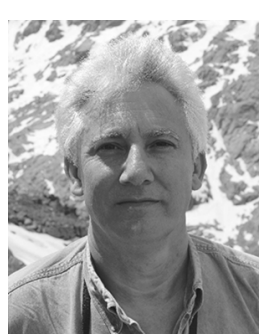

Francisco Medina (M'90-SM'01-F'10) was born in Puerto Real, Cádiz, Spain, in November 1960.

$\mathrm{He}$ received the Licenciado and Ph.D. degrees from the University of Sevilla, Seville, Spain, in 1983 and 1987, respectively, both in physics. He is currently a Professor of electromagnetism with the Department of Electronics and Electromagnetism, University of Sevilla, Seville, Spain, and Head of the Microwaves Group. His research interest includes analytical and numerical methods for planar structures, anisotropic materials, and artificial media modeling. He has coauthored more than 130 book chapters and journal papers on those topics as well as more than 260 conference contributions.

Dr. Medina acts as reviewer for more than 40 IEEE, IEE, AIP and IoP journals and has been a member of the TPCs of a number of local and international conferences. He is now Associate Editor of the International Journal of Microwave and Wireless Technologies. 